\title{
EXTENDING A CAMPUS NETWORK WITH REMOTE BUBBLES USING IPSEC
}

\author{
Aurélien Bonnet \\ Université catholique de Louvain, Dept INGI \\ Place Ste-Barbe, 2, 1348 Louvain-la-Neuve, Belgium \\ ab@info.ucl.ac.be
}

\author{
Marc Lobelle \\ Université catholique de Louvain, Dept INGI \\ Place Ste-Barbe, 2, 1348 Louvain-la-Neuve, Belgium \\ ml@info.ucl.ac.be
}

\begin{abstract}
There is an increasing demand for high speed remote connections (e.g. ADSL, cable, etc.) to university networks both from students and staff members. In many instances, the need is to connect not a single computer but a remote subnetwork and to be able to have not only clients but also servers on the remote subnetwork (for instance to access files on computers on the remote network from workstations in the university). We present here a solution to meet these needs through a simple dial-up-like connection to which the access provider allocates only a single temporary IP address to a single remote machine. The solution allows remote networks to be started anytime like little bubbles and be integrated dynamically into the big bubble that is the university network. It is based on the use of IPSec, DHCP and address translation. Beside providing confidentiality, this allows allocating dynamically IP addresses in the range of the university to computers in the remote bubble, and binding them to permanent DNS names in the domain of the university. After configuration, the computers in the remote subnetwork appear as if they were located inside the university. The solution can, of course, be applied to any organization.
\end{abstract}

Keywords: Remote networks, Virtual private networks, IPSec, DHCP, NAT, RSIP. 


\section{Introduction: Remote access to university networks}

New learning technologies intensively use computers and computer networks. Universities have to provide an increasing number of workstations and personal computers to their students. All these computers are connected to the university network, and through this, to the Internet.

On the other hand, an increasing number of students own or have access to a computer at home or in their residence near the university. In many instances, more than one computer is available: when a new computer is bought, the old one is rarely discarded, but it is rather restricted to the less demanding applications (E-mail, web access, word processing), so that several members of a household can be simultaneously working on computers. In multi-room flats used as student accomodation, several computers are also often available.

However, many learning tasks cannot be performed at home because they require permanent or quasi permanent access to servers of the university, or simply to the Internet. Other activities are only possible with computers that are part of the university network, because of, for instance, software licensing restrictions.

The availability of high speed remote connections (ADSL, Cable) makes it possible to have at home the same kind of access speed as in one's office or in a room of workstations. However, all the other restrictions of dial-up connections remain since remote users get only one dynamically allocated IP address in the range of the provider.

In this paper, we shall discuss how to connect a remote subnetwork when the provider allocates only one dynamic IP address to it. The purpose is to make the remote subnetwork, called remote bubble, really look like part of the university network, with IP addresses belonging to this network. These addresses are centrally and dynamically allocated (central and dynamic allocation avoids wasting IP addresses). As far as we know, this problem has never been addressed before.

\section{Connection of a subnetwork through a single IP address: state of the art}

There are different solutions to connect subnetworks through a single IP address. The first is to use NAT/PAT (Network Address Translation and Port Address Translation); software as well or hardware products (soho routers) implementing this solution are available. They are often based on Linux IP masquerading. Other solutions use tunneling protocols to build a Virtual Private Network. 


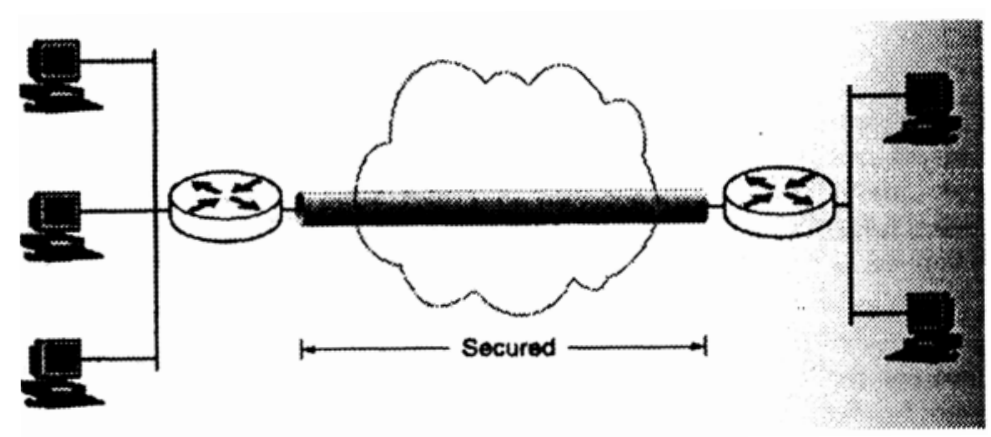

Figure 1. A VPN across the Internet

\subsection{IP Masquerading}

IP Masquerade is a Linux networking function similar to one-to-many NAT (Network Address Translation) found in many firewalls and network routers. For example, if a Linux host, called the gateway, is connected to the Internet, the IP Masquerade feature allows "client" applications on other computers connected to the internal interface of this gateway and using non-routable IP addresses to reach the Internet.

This system allows a set of machines to invisibly access the Internet through the gateway. To other machines on the Internet, all this traffic will appear to be from or to the gateway (for more information see [Ran00]). Not even all "client" applications work with this scheme FTP). It is therefore often complemented with specified application level gateway programs.

\subsection{Building Virtual Private Networks}

The problem that VPNs are trying to solve is that of letting two networks communicate securely when the only connection between them is over a third network which they don't trust. VPNs use a gateway between each of the communicating networks and the untrusted network. Most of the current VPN packages use tunneling to create a private network. The principle of tunneling is to encapsulate a packet within a packet.

The gateway machines can encrypt packets entering the untrusted net and decrypt packets leaving it, creating a secure tunnel through it (see figure 1). 


\subsubsection{Simple tunneling protocol. Most of the current oper-} ating system can enable simple tunnels between two gateways (without any authentication or encryption: the tunnel is thus not secure). This system is very simple. Gateways on each network encapsulate packets destined to the distant network in a packet destined to the remote gateway. But this solution is limited to static IP addresses on gateways. In our context, IP addresses of gateways will be dynamically allocated by the provider. So we need to authenticate these gateways dynamically and by other mean than its IP address.

\subsubsection{The IPSec Protocol and its use in Virtual Private}

Networks . IPSec is a mechanism for adding security to IP and upperlayer protocols. It can protect traffic between hosts, between network security gateways (routers, firewalls,..) and between hosts and security gateways. IPSec hosts and gateways are authenticated by cryptographic technics independantly of their IP addresses, which can be allocated dynamically.

IPSec defines two different protocols: The Encapsulating Security Payload (ESP), and the Authentication Header (AH). AH provides proofof-data-origin, data integrity and antireplay protection on received packets. ESP provides, in addition, data confidentiality and limited traffic flow confidentiality.

More information on the IPSec protocol, can be found in [DH99], [RFC2401], which defines the base architecture upon which all implementation are built, [RFC2402] which explain AH functionalities, and [RFC2406] which talks about ESP.

The VPN can be built by deploying IPSec gateways. The protected network to which access is controlled is on one side of the gateway; the unsafe and unsecured network (usually Internet) is on the other. IPSec must be used in tunnel mode between the gateways, because the VPN is protecting the traffic between two different networks.

\section{Using IPSec VPNs to connect remote bubbles to a university network}

We want to integrate computers at home (or in student residences) transparently in the university network. The homes or the residences are connected to a provider and obtain from it a single temporary IP address: this IP address may be different each time they connect. Several uses of this single temporary IP address are possible:

- One single computer is connected to the modem (ASDL/Cable), and uses the temporary IP address. This solution is the easiest, 


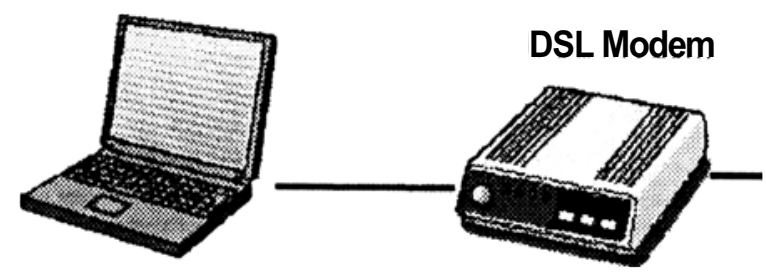

a.b.c.d Provider address

Figure 2. Single connection with a DSL Modem

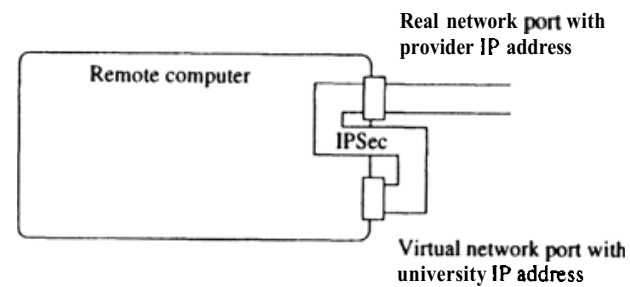

Figure 3. Using IPSec to add an address of the university network to a remote computer

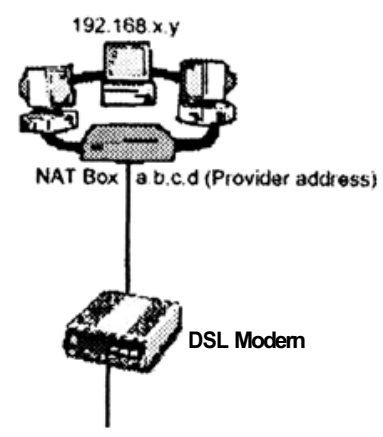

Figure 4. Architecture of a NAT network 


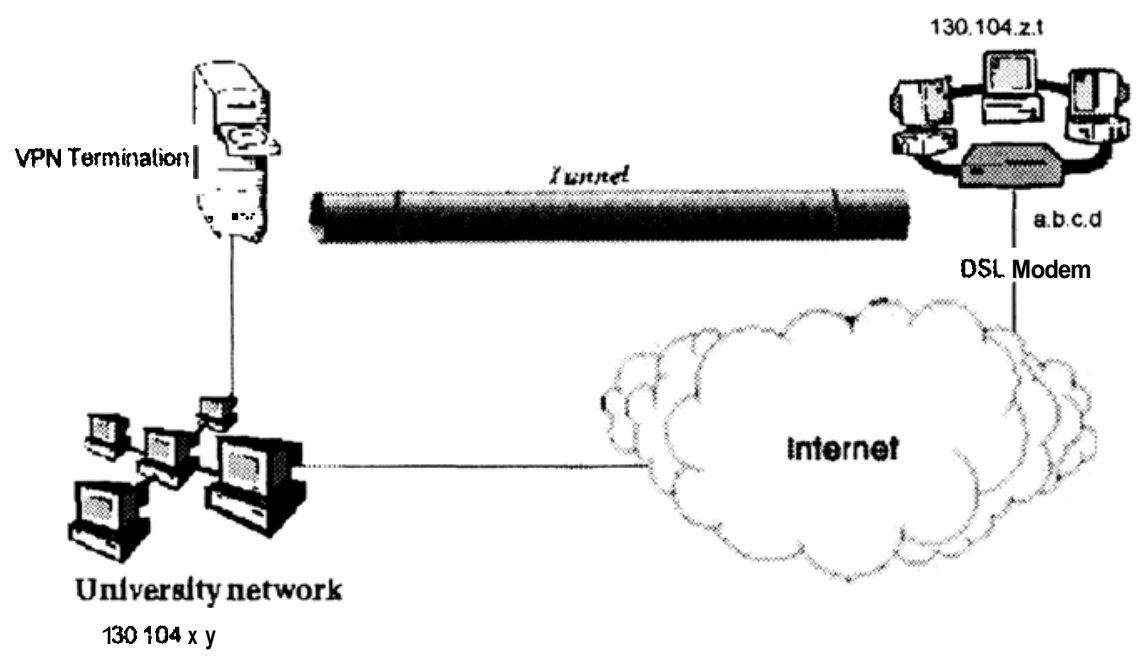

Figure 5. Remote bubble with IPSec

but the computer appears with a provider address, not an address of the university network, as requested (see figure 2). To overcome this problem, an internal address of the university network could be allocated to a virtual interface of the computer and routed through an IPSec VPN across the provider network to a gateway in the university (See figure 3 ). This is actually a particular case of the third situation.

- One computer is a gateway, uses IP masquerading, and connects a subnetwork to the Internet (a dedicated NAT box is equivalent to this. See figure 4). This solution allows to connect more than one computer through a connection with a single routable IP address. It is integrated in Linux, free, and easy to build (several companies market black-boxes with this kind of functionality), but the machines in the subnetwork are not accessible from the Internet (they can only be clients, not servers), and some protocols are not compatible with it (H323, IPSec,...).

- One virtual private network is set up between a gateway to the remote subnetwork and a gateway to the university. This solution doesn't impose any constraint on the address allocation policy in the remote subnetwork: the computers of the remote subnetwork 
can have routable IP addresses. This solution is the only one allowing "remote bubbles" to merge transparently in the university network (see figure 5). That's why we will develop this solution in this section.

\subsection{Requirements of the VPN}

The VPN used to connect the remote subnetwork to the university network must meet the following requirements:

- We want to be able to decide from within the university what addresses will be allocated to the computers on the subnetwork. These addresses may be non routable or routable (for instance taken from the university range).

- We want also to be able to allocate these addresses dynamically but to be bound to permanent domain names. We will not discuss this last aspect in this paper.

- We want the remote network to merge automatically in the university network when the gateway is started. The computers on the remote network must then be undiscernible by third parties from computers located inside the university.

- We want the same security beween computers in the remote network and other computers in the same department of the university as between computers in this department.

\subsection{SubNetwork with IPSec Gateways}

Using an IPSec tunnel between the gateway to the remote subnetwork (let's call it "Hawser") and a gateway in a department of the university (let's call it "Bollard") already allows to meet all but the second of the above requirements (see figure 6).

- The computers of the subnetwork are logically neighbours of the other end of the tunnel.

- IPSec is a standard protocol available for any decent platform.

- IPSec authentication of the gateway is not based on its IP address that can be dynamically allocated by the provider.

- IPSec provides the required security. If confidentiality is needed, ESP can be used, otherwise AH is sufficient. If security is definitely not a problem (for instance if the ADSL links are connected 


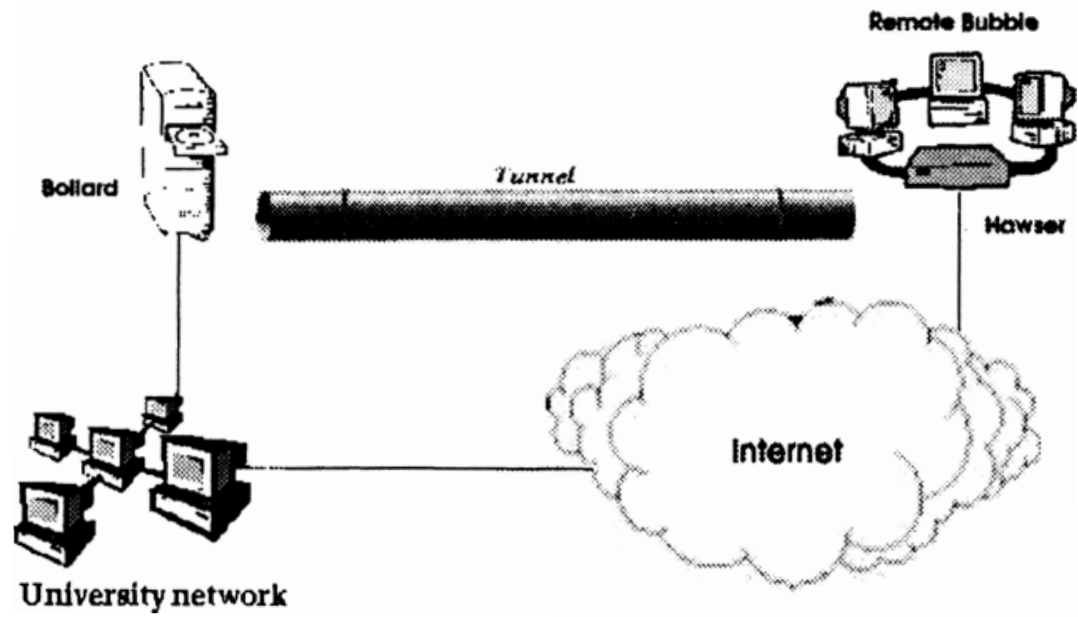

Figure 6. Architecture of the solution

directly by the provider to the university through a VPN instead through the public Internet), then AH with null encoding can be used. The choice is a matter of balance between security and performance. Benchmarks will tell us if providing less than maximum security makes any difference from a performance point of view.

Meeting all these requirements is not straightforward. Let us look more closely at what should happen when Hawser boots:

- Hawser boots.

- Hawser logs into the provider network and gets a dynamic provider address from, say, a radius server.

- Hawser calls Bollard and sets up an IPSec tunnel.

All the packets destined to the subnetwork will be routed through Bollard and Hawser, and all external packets from the subnetwork will be routed through Hawser and Bollard.

\section{Dynamic allocation and address translation}

The Dynamic Host Configuration Protocol (CHCP) automates the process of configuring devices on IP networks. DHCP performs many of 
the functions a network administrator could carry out manually when connecting a new computer to a network (see [DL99]). With DHCP relay agents, remote machines can also be configured. We decided to use the DHCP protocol with relay agents to configure the differents subnetworks for differents reasons:

- addresses can be leased temporarily when needed which simplifies network administration of nomadic computers (laptops),

- subnetworks can be created without any administrative overhead for address allocation,

- the network configuration of the computer is easier (most of the parameters are transmitted by the protocol),

- the DHCP protocol is available on many operating systems.

\subsection{The relay agent}

A relay agent is designed to forward DHCP messages between clients and server when the server and the client are not in the same network. When the relay agent receives a client message, it forwards it to the server, and when the server answers to a request, it also uses the relay agent to contact the client. In our solution, the relay agent runs on Hawser, and the DHCP server can run on any machine of the same network as Bollard, but it is easier to put it directly on Bollard.

\subsection{DHCP configuration}

As specified in [RFC2131], the technique is to assign statically a range of addresses (subnetwork class) to each network behind a relay agent. This solution would be the easiest to deploy but it wastes a lot of IP addresses.

With a modified relay agent, another solution would be to assign addresses to the devices on the different subnetworks without regard for their localization. This solution is more economical in IP addresses, but it is more difficult to deploy, since routes must be explicitly configured for each individual device.

When a device asks for a new DHCP configuration, a dedicated IPSec tunnel must be opened between Bollard and Hawser for this new IP address. This will be done by the relay agent, as it has all the required informations. 


\subsection{Address translation}

The solution proposed in the previous paragraph has a problem. All the packets sent by a device on a subnetwork will be routed through Bollard and Hawser, even the packets destined to same remote bubble, as the differents devices are not considered to be on the same virtual subnetwork. The solution we use to avoid this problem is the address translation (More information available on [Dut01]).

Instead of sending address like "a.b.c.d", the DHCP server will send address like "10.b.c.d" with a class A subnetwork mask. Thus all devices in the same remote bubble can see each other. Hawser will have to "NAT" (translate address) 10.b.c.d and a.b.c.d for incoming and outgoing messages. Devices in other bubbles are known with their addresses allocated and not "10.b.c.d".

\subsection{Summary}

When a new computer is started on a remote bubble:

- the computer boots.

- it broadcasts a DHCP request.

- Hawser forwards the request to Bollard (assuming it is the DHCP server).

- Bollard replies to the request with the address 10.b.c.d

- Hawser forwards the reply to the computer, starts address translation between 10.b.c.d and a.b.c.d, and enables the tunnel for the address a.b.c.d

- the computer is connected to the network.

When a computer uses the network:

- if the address is local (10.x.x.x) it connects directly the specified computer, else it sends the packet to Hawser, which it considers as its default router.

- Hawser exchanges the source address (10.b.c.d to a.b.c.d)

- Hawser sends the new packet to Bollard through the tunnel.

- Bollard routes the packet it receives like a normal packet.

When a computer wants to connect or reply to a computer on a remote bubble: 
- the packet is sent to Bollard.

- Bollard sends it to the Hawser concerned (several remote bubbles can be attached to the same Bollard via different Hawser) through a tunnel.

- Hawser translates the destination address (a.b.c.d to 10.b.c.d).

- Hawser forwards the packet to the computer concerned in the remote bubble.

\section{4. $\quad$ Related Works}

We did not find any other published paper on the problem of setting up a remote subnetwork connected with a single temporary address allocated by a provider and using addresses belonging to a main network and dynamically allocated by a server in this network. However, results has been published about closely related problems.

Realm Specific IP (RSIP) is a new protocol, designed as an alternative to NAT that preserves end to end packet integrity. RSIP allocates on demand in a network A, addresses belonging to another network B. The gateway between networks $\mathrm{A}$ and $\mathrm{B}$ has a pool of IP addresses of $\mathrm{B}$ and will allocate them to hosts in A (called RSIP hosts) when requested. Routes or tunnels must be provided in A for these RSIP hosts. They can be considered as if they were really on the network B.

The advantage of this solution over NAT is that nothing in the packet is modified. The drawback is that a driver is needed in each RSIP host of the subnetwork.

RSIP was designed to solve a different problem than the one addressed in this paper. However, it provides a similar service with different mean. The main diference of the service provided by RSIP and the service provided in our solution is that, since the RSIP server is on the gateway, the range of addresses allocated to each remote subnetwork must be allocated statically. Since our solution uses a central server, addresses can be distributed on demand to several remote networks, which is a more efficient allocation technique.

On the other hand, RSIP preserves the end to end integrity of packets while our solution uses NAT coupled with DHCP which means that packets are modified in the path. The price RSIP has to pay for this advantage is to add some software to each host in the subnetwork.

More Information on RSIP can be found in [RSIP-PROTO]. 


\section{Conclusion}

The paper identifies a new problem, which is to make a remote subnetwork, called remote bubble, really look like merged in another network, with IP addresses belonging to this network centrally and dynamically allocated.

It proposes a solution based on existing protocols (IPSec, DHCP, NAT). Coupled DHCP relay and NAT protocol engines provide the hosts of the remote bubble with addresses from the main network. IPSec allows the gateway itself to have a dynamic IP address and hide the contents of packets on the way between the remote bubble and the main network. The situation of the hosts in the remote bubble is exactly the same (except for communications delays) as if they were located inside the main network.

The solution we propose presents many advantages. In addition to secure communication, with the creation of an IPSec tunnel between the remote bubble and the university network, its dynamic configuration allows to manage easily many bubbles of any size, without wasting IP addresses.

The administration of a bubble is quite simple: all the computers plugged behind the gateway (Hawser) will be automatically on the university network.

\section{Acknowledgements}

This work has been performed in the framework of the SIN project (Secure IP Networking) supported by grant FIRST 981/3749 by MRWDGTRE ("Travaux réalisés avec l'aide du Ministère de la Règion Wallonne, en application du programme de formation et d'impulsion à la recherche scientifique et technologique").

The authors express their thanks to the anonymous reviewers for their useful comments.

\section{References}

[Ran00] Ranch, D. (2000). Linux IP Masquerading HOWTO. Technical report. http://www.linuxdoc.org/HOWTO/IP-Masquerade-HOWTO.html.

[DH99] Doraswamy, N.; Harkins, D. (1999). IPSec : The New Security Standard for the Internet, Intranets and Virtual Private Network. Prentice Hall PTR.

[RFC2401] Kent, S.; Atkinson, R. (1998). Security Architecture for the Internet Protocol. Request for Comments 2401. Network Working Group.

[RFC2402] Kent, S.; Atkinson, R. (1998). IP Authentication Header (AH). Request for Comments 2402. Network Working Group.

[RFC2406] Kent, S.; Atkinson, R. (1998). IP Encapsulating Security Payload (ESP). Request for Comments 2406. Network Working Group. 
[RFC2131] Droms, R. (1997). Dynamic Host Configuration Protocol (DHCP). Request for Comments 2131. Network Working Group.

[DL99] Droms, R.; Lemon, T. (1999). The DHCP Handbook. Macmillan Technical Publishing.

[Dut01] Dutcher, B. (2001). The NAT Handbook . Wiley Computer Publishing.

[RSIP-PROTO] Borella M.; Grabelsky D.; Taniguchi K. (2000). Realm Specific IP: Protocol Specification. Draft. Network Working Group. 